\title{
Enhancing reaction systems: a process algebraic approach
}

\author{
Linda Brodo ${ }^{1 \star[0000-0002-4455-2419]}$, Roberto Bruni ${ }^{20000-0002-7771-4154]}$, and \\ Moreno Falaschi ${ }^{3[0000-0002-6659-3828]}$ \\ 1 Dipartimento di Scienze economiche e aziendali, Università di Sassari, Italy \\ brodo@uniss.it. \\ 2 Dipartimento di Informatica, Università di Pisa, Italy bruni@di.unipi.it. \\ 3 Dipartimento di Ingegneria dell'Informazione e Scienze Matematiche \\ Università di Siena, Italy moreno.falaschi@unisi.it
}

\begin{abstract}
In the area of Natural Computing, reaction systems are a qualitative abstraction inspired by the functioning of living cells, suitable to model the main mechanisms of biochemical reactions. This model has already been applied and extended successfully to various areas of research. Reaction systems interact with the environment represented by the context, and pose problems of implementation, as it is a new computation model. In this paper we consider the link-calculus, which allows to model multiparty interaction in concurrent systems, and show that it allows to embed reaction systems, by representing the behaviour of each entity and preserving faithfully their features. We show the correctness and completeness of our embedding. We illustrate our framework by showing how to embed a lac operon regulatory network. Finally, our framework can contribute to increase the expressiveness of reaction systems, by exploiting the interaction among different reaction systems.
\end{abstract}

Keywords: Process algebras · Reaction systems · Multi-party interaction.

\section{Introduction}

Natural Computing is an emerging area of research which has two main aspects: human designed computing inspired by nature, and computation performed in nature. Reaction Systems (RSs) [9] are a rewriting formalism inspired by the way biochemical reactions take place in living cells. This theory has already shown to be relevant in several different fields, such as computer science [17, biology [2]15]13], and molecular chemistry [18]. Reaction Systems formalise the mechanisms of biochemical systems, such as facilitation and inhibition. As a qualitative approximation of the real biochemical reactions, they consider if a necessary reagent is or not present, and likewise they consider if an inhibiting molecule is or not present. The possible reactants and inhibitors are called

\footnotetext{
* corresponding author
} 
'entities'. RSs model in a direct way the interaction of a living cell with the environment (called 'context'). However, two RSs are seen as independent models and do not interact.

In this paper, we present an encoding from RSs, to the open multiparty process algebra cCNA $A^{4}$ a variant of the link-calculus [5]8] without name mobility. This formalism allows several processes to synchronise and communicate altogether, at the same time, with a new communicating mechanism based on links and link chains. Our initial motivation for introducing this mechanism was to encode Mobile Ambients 12, obtaining a much stronger operational correspondence than any one available in the literature, such as the one in 10. This allowed us to easily encode calculi for biology equipped with membranes, as in [6. Process calculi have been used successfully to model biological processes, see 4 for a recent survey. We illustrate our embedding by means of some simple basic examples, and then we consider a more complex example, by modeling a RS representing a regulatory network for lac operon, presented in [15. We also show that our embedding preserves the main features of RSs, and prove its correctness and completeness. Our main contributions are as follows:

- the behaviour of the context, for each single entity, can be specified in a recursive way as an ordinary process;

- our translation results in a cCNA system where from each state only one transition can be generated, thus the cCNA computation is fully deterministic as that of the encoded RS;

- we can express the behaviour of entity mutation, in such a way that the mutated entity $s^{\prime}$ can take part to only a subset of rules requiring entity $s$;

- with a little coding effort, two RSs can communicate; i.e. a subset of those entities that the context can provide, are then provided by a second RS.

The main drawback of our proposal, is that the cCNA translation is verbose. Nevertheless it is clear that our translation can be automatised by means of a proper front-end in an implementation of the link-calculus.

As we have remarked, in our translation, Reaction Systems get the ability to interact between them in a synchronized manner. This interaction is not foreseen in the basic RS framework, as it can only happen with the context. By exploiting recursion, the kind of interactions which can be defined can be complex and expressive. Example 3 and more in general the discussion in Section 6 show that the interaction between RSs can help to model new scenarios.

Structure of the paper. Section 2 describes RSs and their semantics (interactive processes). Section 3 describes briefly the cCNA process algebra and its operational semantics. Section 4 defines the embedding of RSs in cCNA processes and shows some simple examples to illustrate it. Section 5 shows a more complex example taken from the literature on RSs and illustrates a lac operon. Section 6 presents some features and advantages of our embedding for the compositionality of RSs. Finally, Section 7 discusses future work and concludes.

\footnotetext{
${ }^{4}$ After 'chained Core Network Algebra'.
} 


\section{Reaction Systems}

Natural Computing is concerned with human-designed computing inspired by nature as well as with computation taking place in nature. The theory of Reaction Systems [9] was born in the field of Natural Computing to model the behaviour of biochemical reactions taking place in living cells. Despite its initial aim, this formalism has shown to be quite useful not only for modeling biological phenomena, but also for the contributions given to computer science [17, theory of computing, mathematics, biology [2]15]13], and molecular chemistry [18]. Here we briefly review the basic notions of RSs, see [9] for more details.

The mechanisms that are at the basis of biochemical reactions and thus regulate the functioning of a living cell, are facilitation and inhibition. These mechanisms are reflected in the basic definitions of Reaction Systems.

Definition 1 (Reaction). A reaction is a triplet $a=(R, I, P)$, where $R, I, P$ are finite, non empty sets and $R \cap I=\emptyset$. If $S$ is a set such that $R, I, P \subseteq S$, then a is a reaction in $S$.

The sets $R, I, P$ are also written $R_{a}, I_{a}, P_{a}$ and called the reactant set of $a$, the inhibitor set of $a$, and the product set of $a$, respectively. All reactants are needed for the reaction to take place. Any inhibitor blocks the reaction if it is present. Products are the outcome of the reaction. Also, $R_{a} \cup I_{a}$ is the set of the resources of $a$ and $\operatorname{rac}(S)$ denotes the set of all reactions in $S$. Because $R$ and $I$ are non empty, all products are produced from at least one reactant and every reaction can be inhibited in some way. Sometimes artificial inhibitors are used that are never produced by any reaction. For the sake of simplicity, and without loss of generality, in some examples, we will allow $I$ to be empty.

Definition 2 (Reaction System). A Reaction System (RS) is an ordered pair $\mathcal{A}=(S, A)$ such that $S$ is a finite set, and $A \subseteq \operatorname{rac}(S)$.

The set $S$ is called the background set of $\mathcal{A}$, its elements are called entities, and they represent molecular substances (e.g., atoms, ions, molecules) that may be present in the states of a biochemical system. The set $A$ is the set of reactions of $\mathcal{A}$. Since $S$ is finite, so is $A$ : we denote by $|A|$ the number of reactions in $A$.

Definition 3 (Reaction Result). Given a finite set of entities $S$, let $T \subseteq S$.

1. Let $a \in \operatorname{rac}(S)$ be a reaction. Then a is enabled by $T$, denoted by en $(T)$, if $R_{a} \subseteq T$ and $I_{a} \cap T=\emptyset$.

2. Let $a \in \operatorname{rac}(S)$ be a reaction. The result of a on $T$, denoted by $\operatorname{res}_{a}(T)$, is defined by: $\operatorname{res}_{a}(T)=P_{a}$ if en $(T)$, and res $s_{a}(T)=\emptyset$ otherwise.

3. Let $A \subseteq \operatorname{rac}(S)$ be a finite set of reactions. The result of $A$ on $T$, denoted by $\operatorname{res}_{A}(T)$, is defined by: $\operatorname{res}_{A}(T)=\bigcup_{a \in A} \operatorname{res}_{a}(T)$.

The theory of Reaction Systems is based on the following assumptions.

- No permanency. An entity of a set $T$ vanishes unless it is sustained by a reaction. This reflects the fact that a living cell would die for lack of energy, without chemical reactions. 
- No counting. The basic model of RSs is very abstract and qualitative, i.e. the quantity of entities that are present in a cell is not taken into account.

- Threshold nature of resources. From the previous item, we assume that either an entity is available and there is enough of it (i.e. there are no conflicts), or it is not available at all.

The dynamic behaviour of a RS is formalized in terms of interactive processes.

Definition 4 (Interactive Process). Let $\mathcal{A}=(S, A)$ be a $R S$ and let $n \geq 0$. An n-step interactive process in $\mathcal{A}$ is a pair $\pi=(\gamma, \delta)$ of finite sequences s.t. $\gamma=\left\{C_{i}\right\}_{i \in[0, n]}$ and $\delta=\left\{D_{i}\right\}_{i \in[0, n]}$ where $C_{i}, D_{i} \subseteq S$ for any $i \in[0, n], D_{0}=\emptyset$, and $D_{i}=\operatorname{res}_{\mathcal{A}}\left(D_{i-1} \cup C_{i-1}\right)$ for any $i \in[1, n]$.

Living cells are seen as open systems that continuously react with the external environment, in discrete steps. The sequence $\gamma$ is the context sequence of $\pi$ and represents the influence of the environment on the Reaction System. The sequence $\delta$ is the result sequence of $\pi$ and it is entirely determined by $\gamma$ and $A$. The sequence $\tau=W_{0}, \ldots, W_{n}$ with $W_{i}=C_{i} \cup D_{i}$, for any $i \in[0, n]$ is called a state sequence. Each state $W_{i}$ in a state sequence is the union of two sets: the context $C_{i}$ at step $i$ and the result of the previous step.

For technical reasons, we extend in a straightforward way the notion of an interactive process to deal with infinite sequences.

Definition 5 (extended interactive process). Let $\mathcal{A}=(S, A)$ be a $R S$, and let $\pi=(\gamma, \delta)$ be an n-step interactive process, with $\gamma=\left\{C_{i}\right\}_{i \in[0, n]}$ and $\delta=$ $\left\{D_{i}\right\}_{i \in[0, n]}$. Then, let $\pi^{\prime}=\left(\gamma^{\prime}, \delta^{\prime}\right)$ be the extended interactive process of $\pi=$ $(\gamma, \delta)$, defined as $\gamma^{\prime}=\left\{C_{i}^{\prime}\right\}_{i \in \mathbb{N}}, \delta^{\prime}=\left\{D_{i}^{\prime}\right\}_{i \in \mathbb{N}}$, where $C_{j}^{\prime}=C_{j}$ for $j \in[0, n]$ and $C_{j}^{\prime}=\emptyset$ for $j>n, D_{0}^{\prime}=D_{0}$ and $D_{j}^{\prime}=\operatorname{res}_{A}\left(D_{j-1}^{\prime} \cup C_{j-1}^{\prime}\right)$ for $j \geq 1$.

The next example shows that the functioning of the interacting processes is deterministic, once the context is fixed.

Example 1. Let $\mathcal{A}=(S, A)$ be a RS with $S=\left\{s_{1}, s_{2}, s_{3}, s_{4}\right\}$, and $A=\left\{a_{1}, a_{2}\right\}$, where $a_{1}=\left(\left\{s_{1}\right\},\left\{s_{3}\right\},\left\{s_{1}\right\}\right), a_{2}=\left(\left\{s_{2}\right\},\left\{s_{4}\right\},\left\{s_{2}\right\}\right)$. Now, we show the first three steps of an interacting process $\pi=(\gamma, \delta)$ where the context provides entities as follows: $\gamma=\left\{C_{0}=\left\{s_{1}, s_{2}\right\}, C_{1}=\left\{s_{3}\right\}, C_{2}=\left\{s_{1}, s_{4}\right\}, C_{3}=\emptyset, C_{4}, \ldots, C_{n}\right\}$. Initally $D_{0}=\emptyset$ and from $C_{0} \cup D_{0}=\left\{s_{1}, s_{2}\right\}$ we get to $C_{1} \cup D_{1}=\left\{s_{1}, s_{2}, s_{3}\right\}$ by applying $a_{1}, a_{2}$ (they both are enabled); after that we get to $C_{2} \cup D_{2}=$ $\left\{s_{1}, s_{2}, s_{4}\right\}$, by applying $a_{2}$ only ( $a_{1}$ is not enabled); finally we get to $C_{3} \cup D_{3}=$ $\left\{s_{1}\right\}$ by applying $a_{1}$ ( $a_{2}$ is not enabled). Thus $\delta=\left\{D_{0}=\emptyset, D_{1}=\left\{s_{1}, s_{2}\right\}, D_{2}=\right.$ $\left.\left\{s_{2}\right\}, D_{3}=\left\{s_{1}\right\}, D_{4}, \ldots, D_{n}\right\}$. We remark that at every state $C_{i} \cup D_{i}$ all the reactions which are enabled are applied, so the computation is deterministic.

\section{Chained CNA (cCNA)}

In this section we introduce the syntax and operational semantics of a variant of the link-calculus [5], the cCNA (chained CNA), where the prefixes are link chains. 
Link Chains. Let $\mathcal{C}$ be the set of channels, ranged over by $a, b, \ldots$, and let $\mathcal{N}=$ $\mathcal{C} \cup\{\tau\} \cup\{\square\}$ be the set of actions, ranged over by $\alpha, \beta, \ldots$, where the symbol $\tau$ denotes a silent action, while the symbol $\square$ denotes a virtual (non-specified) action. A link is a pair $\ell={ }^{\alpha} \backslash_{\beta}$; it is solid if $\alpha, \beta \neq \square$; the link ${ }^{\square} \square$ is called virtual; the link ${ }^{\tau} \backslash_{\tau}$ is called silent. A link is valid if it is solid or virtual. A link chain is a finite sequence $v=\ell_{1} \ldots \ell_{n}$ of valid links $\ell_{i}={ }^{\alpha_{i}} \backslash_{\beta_{i}}$ such that:

1. for any $i \in[1, n-1], \begin{cases}\beta_{i}, \alpha_{i+1} \in \mathcal{C} & \text { implies } \beta_{i}=\alpha_{i+1} \\ \beta_{i}=\tau & \text { iff } \alpha_{i+1}=\tau\end{cases}$

2. $\exists i \in[1, n] . \ell_{i} \neq \square_{\square}$.

A link chain whose links are silent is also called silent. Virtual links ${ }^{\square} \backslash$ represent missing elements of a chain. The equivalence $\boldsymbol{}$ models expansion and contraction of virtual links to adjust the length of a link chain.

Definition 6 (Equivalence $\$ ). We let $\gg$ be the least equivalence relation over link chains closed under the axioms (whenever both sides are well defined):

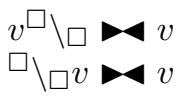

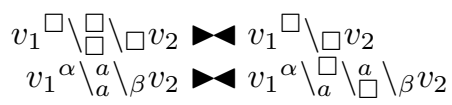

Two link chains of equal length can be merged whenever each position occupied by a solid link in one chain is occupied by a virtual link in the other chain and solid links in adjacent positions match. Positions occupied by virtual links in both chains remain virtual. Merging is denoted by $v_{1} \bullet v_{2}$. For example, given $v_{1}={ }^{a} \backslash{ }_{b} \backslash \square \backslash \square$ and $v_{2}=\square \backslash_{\square}^{b} \backslash \backslash \backslash \backslash \square$ we have $v_{1} \bullet v_{2}={ }^{a} \backslash_{b}^{b} \backslash_{c} \backslash \square$.

Some names in a link chain can be restricted as non observable and transformed into silent actions $\tau$. This is possible only if they are matched by some adjacent link. Restriction is denoted by $(\nu a) v$. For example, given $\left.v={ }^{a} \backslash_{b}^{b}\right\rangle_{c}^{\square} \backslash \square$ we have $(\nu b) v={ }^{a} \backslash_{\tau}^{\tau} \backslash_{c}^{\square} \backslash \square$.

Syntax. The cCNA processes are generated by the following grammar:

$$
P, Q::=\sum_{i \in I} v_{i} \cdot P_{i}|P| Q|(\nu a) P| P[\phi] \mid A
$$

where $v_{i}$ is a link chain, $\phi$ is a channel renaming function, and $A$ is a process identifier for which we assume a definition $A \triangleq P$ is available in a given set $\Delta$ of (possibly recursive) process definitions. We let $\mathbf{0}$, the inactive process, denote the empty summation.

The syntax of cCNA extends that of CNA $[8$ by allowing to use link chains as prefixes instead of links. This extension was already discussed in [8] and it preserves all the main formal properties of CNA. For the rest it features nondeterministic choice, parallel composition, restriction, relabelling and possibly recursive definitions of the form $A \triangleq P$ for some constant $A$. Here we do not consider name mobility, which is present instead in the link-calculus. 


$$
\begin{aligned}
& \frac{v \bowtie v_{j} \quad j \in I}{\sum_{i \in I} v_{i} . P_{i} \stackrel{v}{\rightarrow} P_{j}}(\text { Sum }) \quad \frac{P \stackrel{v}{\rightarrow} P^{\prime} \quad(A \triangleq P) \in \Delta}{A \stackrel{v}{\rightarrow} P^{\prime}} \text { (Ide) } \\
& \frac{P \stackrel{v}{\rightarrow} P^{\prime}}{P[\phi] \stackrel{\phi(v)}{\rightarrow} P^{\prime}[\phi]}(\text { Rel }) \quad \frac{P \stackrel{v}{\rightarrow} P^{\prime}}{(\nu a) P \stackrel{(\nu a) v}{\longrightarrow}(\nu a) P^{\prime}} \text { (Res) } \\
& \frac{P \stackrel{v}{\rightarrow} P^{\prime}}{P\left|Q \stackrel{v}{\rightarrow} P^{\prime}\right| Q} \text { (Lpar) } \quad \frac{P \stackrel{v^{\prime}}{\rightarrow} P^{\prime} \quad Q \stackrel{v}{\rightarrow} Q^{\prime}}{P\left|Q \stackrel{v \bullet v^{\prime}}{\rightarrow} P^{\prime}\right| Q^{\prime}}(\text { Com })
\end{aligned}
$$

Fig. 1: SOS semantics of the cCNA (rules (Rel) and (Rpar) omitted).

Semantics. The operational semantics of cCNA is defined in the SOS style by the inference rules in Fig 1 . The rules are reminiscent of those for Milner's CCS and they essentially coincide with those of CNA in [8], except for the presence of prefixes that are link chains instead of single links. Briefly: rule (Sum) selects one alternative and uses, as a label, a possible contraction/expansion $v$ of the link chain $v_{j}$ in the selected prefix; rule (Ide) selects one transition of the process defined by a constant; rule $(R e l)$ renames the channels in the label as indicated by $\phi$; rule (Res) restricts some names in the label (it cannot be applied when $(\nu a) v$ is not defined); rules (Lpar) and (Rpar) account for interleaving in parallel composition; rule ( $\mathrm{Com}$ ) synchronises interactions (it cannot be applied when $v \bullet v^{\prime}$ is not defined). Analogously to CNA, the operational semantics of cCNA satisfies the so called Accordion Lemma: whenever $P \stackrel{v}{\rightarrow} Q$ and $v^{\prime} v$ then $P \stackrel{v^{\prime}}{\rightarrow} Q$. As a matter of notation, we write $P \rightarrow Q$ when $P \stackrel{v}{\rightarrow} Q$ for some silent link chain $v$ and call it a silent transition. Similarly, a sequence of $j$ silent transitions is denoted $P \rightarrow^{j} Q$.

\subsection{Notation for link chains}

Hereafter we make use of some new notations for link chains that will facilitate the presentation of our translation.

Definition 7 (Replication). Let $v$ be a link chain. Its $n$ times replication $v^{n}$ is defined recursively by letting $v^{0}=\epsilon$ (i.e. the empty chain) and $v^{n}=v^{n-1} v$, with the hypothesis that all the links in the resulting link chains match.

For example, the expression $\left({ }^{a} \backslash_{b}^{\square} \backslash \square\right)^{3}$ denotes the chain ${ }^{a} \backslash_{b}^{\square} \backslash_{\square}^{a} \backslash_{b} \backslash_{\square}^{a} \backslash_{b} \backslash \square$. We introduce the half link that will be used in conjunction with the open block of chain to form regular link chains. Let ${ }^{a} \backslash$ denote the half left link of a link ${ }^{a} \backslash_{x}$, and conversely let $\backslash_{a}$ denote the half right link of ${ }^{x} \backslash_{a}$.

Definition 8 (Open block). Let $R$ be a totally ordered, finite set of names. We define an open block as $\left(\prod_{c \in R} \square_{i} \backslash c_{\square}\right)$, where $c_{i}$ and $c_{o}$ are annotated version of the name c, as follows 


$$
\begin{aligned}
& \text { set } \\
& R=\emptyset \\
& R=\{a\} \\
& R=\{a\} \uplus R^{\prime} \text { with } a=\min R \\
& \text { open block expression result } \\
& \left(\prod_{c \in R} \square \backslash c_{i} \backslash \begin{array}{c}
c_{o} \\
\square
\end{array}\right) \quad \epsilon
\end{aligned}
$$

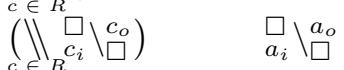

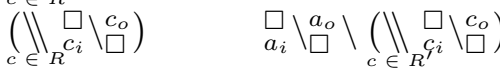

We then combine half links and open blocks to form regular link chains. For example, for $R=\{a, b\}$ the expression $\left(\prod_{c \in R} \bigvee_{R} c_{i} \backslash c_{\square}\right)$ denotes the block of chains

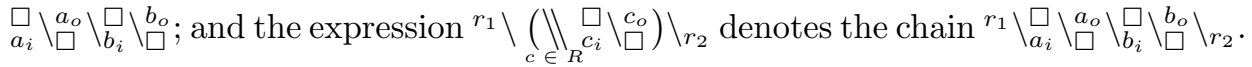

\section{From Reaction Systems to cCNA}

Here we present a translation from Reaction Systems to cCNA. The idea is to define separated processes for representing the behaviour of each entity, each reaction, and for the provisioning of each entity by the context.

Processes for entities. Given an entity $s \in S$, we exploit four different names for the interactions over $s$ : names $s_{i}, s_{o}$ are used to test the presence of $s$ in the system; names $\hat{s}_{i}, \hat{s}_{o}$ are used to test the provisioning of $s$ from the context; names $\tilde{s}_{i}, \tilde{s}_{o}$ are used to test the production of $s$ by some reaction; names $\bar{s}_{i}, \bar{s}_{O}$ are used to test the absence of $s$ in the system; and names $\underline{s}_{i}, \underline{s}_{O}$ are used to test the absence of $s$ from the context. We let $P_{s}$ be the process implementing the presence of $s$ in the system, and $\overline{P_{s}}$ its absence. They are defined below:

$$
\begin{aligned}
& P_{s} \triangleq \sum_{h \geq 0, k \geq 0}\left({ }^{\left(s_{i}\right.} \backslash_{s_{o}} \backslash \square\right)^{h} \hat{s}_{i} \backslash \bigwedge_{\hat{s}_{o}} \backslash \square\left(\tilde{s}_{i} \backslash \breve{s}_{\tilde{s}_{o}} \backslash \square\right)^{k} \cdot P_{s} \\
& + \\
& \sum_{h \geq 0, k \geq 1}\left({ }^{s_{i}} \backslash{ }_{s_{o}} \backslash \square\right)^{h} \underline{s}_{i} \backslash_{\underline{s}_{o}} \backslash \square\left(\tilde{s}_{i} \backslash \bigvee_{\tilde{s}_{o}} \backslash \square\right)^{k} \cdot P_{s} \\
& \sum_{h \geq 0}^{+}\left({ }^{\left(s_{i}\right.} \backslash_{s_{o}} \backslash \square\right)^{h} \underline{s}_{i} \backslash \underline{s}_{o} \cdot \overline{P_{s}}
\end{aligned}
$$

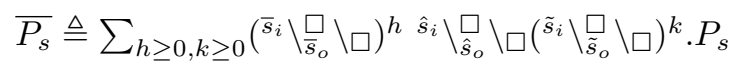

$$
\begin{aligned}
& + \\
& \sum_{h \geq 0, k \geq 1}\left(\bar{s}_{i} \backslash \bar{s}_{o} \backslash \square\right)^{h} \underline{s}_{i} \backslash \underline{\underline{s}}_{o} \backslash \square\left({ }^{\tilde{s}_{i}} \backslash \tilde{\tilde{s}}_{o} \backslash \square\right)^{k} \cdot P_{s} \\
& \sum_{h \geq 0}^{+}\left(\bar{s}_{i} \backslash \bar{s}_{o} \backslash \square\right)^{h} \underline{s}_{i} \backslash \underline{s}_{o} \cdot \overline{P_{s}}
\end{aligned}
$$

The first line of $P_{s}$ accounts for the case where $s$ is tested for presence by $h$ reactions and produced by $k$ reactions, while being provided by the context $\left(\hat{s}_{i} \backslash \hat{s}_{o}\right)$. Thus, $s$ will be present at the next step (the continuation is $P_{s}$ ). Here $h$ and $k$ are not known a priori and therefore any combination is possible. By knowing the number of reactions that test $s$, we can bound the maximum values of $h$ and $k$. The second line accounts for the analogous case where $s$ is not provided by the context $\left(\underline{s}_{i} \underline{\underline{s}}_{o}\right)$. The condition $k \geq 1$ guarantees that $s$ will 
remain present (the continuation is $P_{s}$ ). The third line accounts for the case where $s$ is tested for presence by $h$ reactions, but it is neither produced nor provided by the context. Therefore, in the next step $s$ will be absent in the system (the continuation is $\overline{P_{s}}$ ). Note that in the case of $\overline{P_{s}}$ the test for presence of $s$ in the system is just replaced by the test for its absence. As before, $s$ will be present again in the system if $s$ will be produced (rows 1 and 2 in $\overline{P_{s}}$ code), or if the context will provide it (row 1 in $\overline{P_{s}}$ code).

Processes for reactions. We assume that each reaction $a$ is assigned a progressive number $j$. The process for reaction $a_{j}=\left(R_{j}, I_{j}, P_{j}\right)$ must assert either the possibility to apply the reaction or its impossibility. The first case happens when all its reactants are present (the link ${ }^{s_{i}} \backslash s_{o}$ is requested for any $s \in R_{j}$ ) and all its inhibitors are absent (the link ${ }^{\bar{e}_{i}} \backslash \backslash_{\bar{e}_{o}}$ is requested for any $e \in I_{j}$ ), then the product set is released (the link $\tilde{c}_{i} \backslash_{\tilde{c}_{o}}$ is requested for any $c \in P_{j}$ ). The next case can happen for two reasons: one of the reactants is absent (the link $\bar{s}_{i} \backslash_{\bar{s}_{o}}$ is requested for some $s \in R_{j}$ ) or one of the inhibitors is present (the link ${ }^{e_{i}} \backslash_{e_{o}}$ is requested for some $e \in I_{j}$ ). The process is recursive so that reactions can be applied at any step.

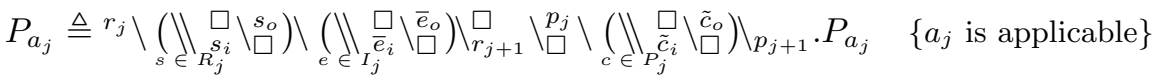

$$
\begin{aligned}
& + \\
& \sum_{s \in R_{j}} r_{j} \backslash \bar{s}_{i} \backslash \stackrel{\bar{s}}{\square} \backslash_{\square}^{\bar{s}_{r_{j+1}}} \backslash_{\square}^{p_{j}} \backslash p_{j+1} \cdot P_{a_{j}} \quad\left\{a_{j} \text { is not applicable }\right\} \\
& + \\
& \sum_{e \in I_{j}} r_{j} \backslash \square_{e_{i}}^{\square} \backslash \stackrel{e_{o}}{\square} \backslash_{r_{i+1}} \backslash \stackrel{p_{j}}{\square} \backslash p_{j+1} . P_{a_{j}} \quad\left\{a_{j} \text { is not applicable }\right\}
\end{aligned}
$$

Formally speaking, the definition of the case when $a_{j}$ is applicable (row 1 ) requires $R_{j}, I_{j}$ and $P_{j}$ to be totally ordered, because open block expressions are used. It is worth noting that the chosen orders are inessential for exploiting $P_{a_{j}}$ in the encoding of a RS, but they are needed to disambiguate its definition. Without loss of generality, we assume that all the entities are enumerated, likewise reactions, so that $R_{j}, I_{j}$ and $P_{j}$ inherit the same order. We exploit names $r_{j}, p_{j}$ to join the chains provided by the application of all the reactions. Channels $r_{j}$ and $r_{j+1}$ enclose the enabling/disabling condition of reaction $a_{j}$. Channels $p_{i}$ and $p_{j+1}$ enclose the links related to the entities produced by $a_{j}$. We will see that all the link chain labels of transitions follow the same schema: first we find all the reactions limited to the reactants and inhibitors (chained using $r_{j}$ channels), then all the supplies by the contexts (chained using $c x t_{j}$ channels, to be introduced next), and finally the products for all the reactions (chained using $p_{j}$ channels). In the following there is an example explaining this schema.

Processes for contexts. For each entity $s \in S$, we introduce another process $C x t_{s}$, participating in each transition and determining whether the entity $s$ is provided by the context or not. As already said, we assume that entities are enumerated and use the names $c x t_{j}$ to concatenate the chains formed by the application of all the contexts. For each entity $s$ with number $j$, at step $n>0$ there are two 
possible behaviours:

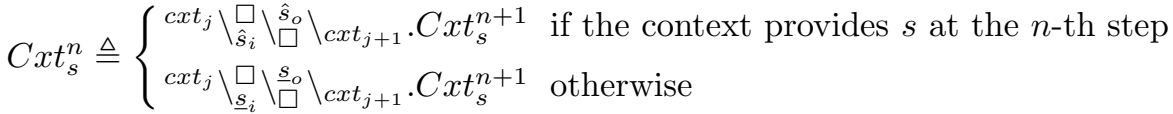

$$
\begin{aligned}
& C x t_{s} \triangleq C x t_{s}^{1}
\end{aligned}
$$

We only consider $C x t_{s}^{n}$ with $n>0$, as the entities such that $s \in C_{0}$ (resp. $s \notin C_{0}$ ) are modeled by the $P_{s}$ processes (resp. $\overline{P_{s}}$ ) in the initial cCNA process. The intrinsic modularity of cCNA allows us to run different context behaviours for the same system by changing the definitions of $C x t_{s}^{n}$.

Definition 9 (Translation). Let $\mathcal{A}=(S, A)$ be a $R S$, and let $\pi=(\gamma, \delta)$ be an extended interactive process in $\mathcal{A}$, with $\gamma=\left\{C_{i}\right\}_{i \in \mathbb{N}}$. We define its cCNA translation $\llbracket \mathcal{A}, \gamma \rrbracket$ as follows:

$$
\llbracket \mathcal{A}, \gamma \rrbracket=(\nu \text { reacts, cxts, ents, prods })\left(\Pi_{s \in C_{0}} P_{s}\left|\Pi_{s \notin C_{0}} \overline{P_{s}}\right| \Pi_{a \in A} P_{a} \mid \Pi_{s \in S} C x t_{s}\right),
$$

with reacts be the set of reaction names $r_{j}$, cxts the set of context names cxt $_{j}$, ents the set of decorated entity names $\left\{s_{i}, s_{o}, \hat{s}_{i}, \hat{s}_{o}, \tilde{s}_{i}, \tilde{s}_{o}, \bar{s}_{i}, \bar{s}_{o}, \underline{s}_{i}, \underline{s}_{o} \mid s \in S\right\}$, and prods be the set of names $p_{j}$ associated to each reaction. In the following, we set names $=$ reacts $\cup$ cxts $\cup$ ents $\cup$ prods. For notational convenience, we fix that $r_{1}=\tau, r_{u+1}=c x t_{1}$ for $u$ the number of reacts, and $c x t_{w+1}=p_{1} p_{u+1}=\tau$ for $w$ the number of entities.

It is important to observe that, for each transition, our cCNA encoding requires all the processes $P_{a}$, with $a \in A$, and $C x t_{s}$ and $P_{s}$, with $s \in S$, be interacting in that transition. This is due to the fact that all the channels $r_{j}, p_{j}$, $c x t_{j}$, and $s_{i}$, and $s_{o}$ are restricted. Each reaction defines a pattern to be satisfied, i.e. each reaction inserts as many virtual links as the number of reactants, inhibitors, and products, as required by the corresponding reaction.

Lemma 1. Let $\mathcal{A}=(S, A)$ be a $R S$ and let $\pi=(\gamma, \delta)$ be an extended interactive process in $\mathcal{A}$. Let $P=\llbracket \mathcal{A}, \gamma \rrbracket$ its cCNA translation. If there exists $P^{\prime}$ such that $t=\left(P \stackrel{(\nu \text { names }) v}{\longrightarrow} P^{\prime}\right)$ is a transition of $P$, then

1. for each reaction $a_{j} \in A$, the corresponding channels $r_{j}$ and $p_{j}$ appear in $v$; for each entity $s_{h} \in S$ (where $h$ is the identifying number of $s$ ), the corresponding channel $s_{h}$ (suitably decorated), and the corresponding channel $c t_{h}$ appear in $v$;

2. for each reaction $a \in A$ and each entity $s \in S$, each virtual link offered by processes $P_{a}$ and $C x t_{s}$ is overlapped by exactly one solid link offered by processes representing entities.

Example 2. Let $\mathcal{A}$ be a RS whose specification contains two entities, $s 1$ and $s 2$, and, among the others, the reaction $a=(\{s 1\},\{\ldots\},\{s 1\})$ that guarantees the persistence of the entity $s 1$ once it is present in the system. Note that we use here a dummy inhibitor (...) which will never be present. Then, we assume an 


\begin{tabular}{|c|c|c|c|c|c|c|c|c|c|c|c|c|}
\hline$\tau$ & $\square$ & $s 1_{o}$ & & $c x t_{1}$ & & $c x t_{2}$ & $\square$ & $\hat{s 2} O$ & $p_{1}$ & $\square$ & $\tilde{s 1} o$ & \\
\hline & $s 1_{i}$ & $\square$ & $r_{2}$ & & $\hat{s 1}_{i}$ & & $\hat{s}_{i}$ & $\square$ & $p_{1}$ & $\tilde{s 1}{ }_{i}$ & $\square$ & $\tau$ \\
\hline
\end{tabular}

Fig. 2: The link chain structure arising from reactions and context processes.

extended interactive process $\pi=(\gamma, \delta)$ where the context $\gamma$ provides $s 1$ and $s 2$. Our translation includes the processes:

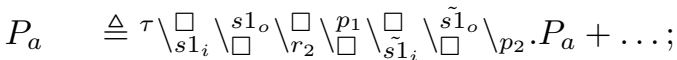

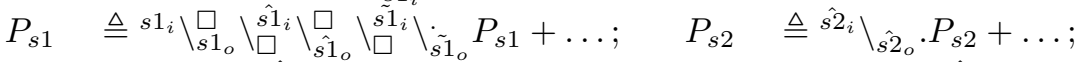

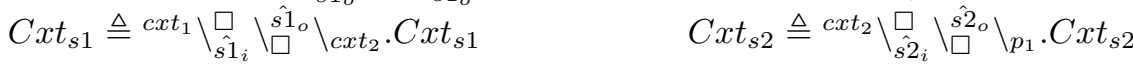

Now, we assume that $s 1$ is in the initial state of $\mathcal{A}$, and in Figure 2 we show the structure of a link chain label related to the execution of a transition of the cCNA system: $(\nu$ names $)\left(P_{s 1}\left|P_{s 2}\right| P_{a}|\ldots| C x t_{s 1} \mid C x t_{s 2}\right)$. The yellow blocks are referred to the processes encoding the reactions $\left(P_{a}\right.$, in our case) and the contexts $\left(C x t_{s 1}\right.$ and $C x t_{s 2}$ ). As the figure puts in evidence, these two kinds of processes determine the structure of the link chain, from end to end, i.e. from the left $\tau$ to the right one. We could say that these processes form the backbone of the interaction. In contrast, the processes encoding the entities $\left(P_{s 1}\right.$, and $P_{s 2}$, in our case) provides the solid links to overlap the virtual links of the backbone.

Example 2 outlines two different roles of the processes defining the translation of an interactive process: those processes encoding the reactions and the context provide the backbone of each transition, whereas the processes encoding the entities provide the resources needed for the communication to take place.

With the next proposition, we analyse the structure of a cCNA process encoding of a reactive process after one step transition. In the following four statements, for brevity, we let $\mathcal{A}=(S, A)$ be a RS, and let $\pi=(\gamma, \delta)$ be an extended interactive process in $A$, with $\gamma=\left\{C_{i}\right\}_{i \in \mathbb{N}}$ and $\delta=\left\{D_{i}\right\}_{i \in \mathbb{N}}$. Moreover, we denote by $\pi^{j}$ the shift of $\pi$ starting at the $j$-th state sequence; formally we let $\pi^{j}=\left(\gamma^{j}, \delta^{j}\right)$ with $\gamma^{j}=\left\{C_{i}^{\prime}\right\}_{i \in \mathbb{N}}, \delta^{j}=\left\{D_{i}^{\prime}\right\}_{i \in \mathbb{N}}$ such that $C_{0}^{\prime}=C_{j} \cup D_{j}, D_{0}^{\prime}=\emptyset$, and $C_{i}^{\prime}=C_{i+j}, D_{i}^{\prime}=D_{i+j}$ for any $i \geq 1$.

Proposition 1 (Correctness 1). Let $P=\llbracket \mathcal{A}, \gamma \rrbracket$ with

$$
P=(\nu \text { names })\left(\Pi_{a \in A} P_{a}\left|\Pi_{s \in S} C x t_{s}^{1}\right| \Pi_{s \in C_{0}} P_{s} \mid \Pi_{s \notin C_{0}} \bar{P}_{s}\right) .
$$

If $P \stackrel{v}{\rightarrow} Q$, then $v$ is a silent action and $Q=\llbracket \mathcal{A}, \gamma^{1} \rrbracket$, namely

$$
Q=(\nu \text { names })\left(\Pi_{a \in A} P_{a}\left|\Pi_{s \in S} C x t_{s}^{2}\right| \Pi_{s \in C_{1} \cup D_{1}} P_{s} \mid \Pi_{s \notin C_{1} \cup D_{1}} \bar{P}_{s}\right) .
$$

Now, we extend the previous result to a series of transitions.

Corollary 1 (Correctness 2). Let $P=\llbracket \mathcal{A}, \gamma \rrbracket$ and $j \geq 1$. If there exists $Q$ such that $P \rightarrow^{j} Q$, then $Q=\llbracket \mathcal{A}, \gamma^{j} \rrbracket$. 
Conversely, we prove that the $c$ CNA process $\llbracket \mathcal{A}, \gamma \rrbracket$ can simulate all the evolutions of the underlying interactive process.

Proposition 2 (Completeness 1). $\llbracket \mathcal{A}, \gamma \rrbracket \rightarrow \llbracket \mathcal{A}, \gamma^{1} \rrbracket$.

Now, we extend the previous result to a series of transitions.

Corollary 2 (Completeness 2). $\llbracket \mathcal{A}, \gamma \rrbracket \rightarrow^{j} \llbracket \mathcal{A}, \gamma^{j} \rrbracket$.

\section{Example: lac operon}

In this section we present the encoding of a RS example taken from [15].

\subsection{The lac operon}

An operon is a cluster of genes under the control of a single promoter. The lac operon is involved in the metabolism of lactose in Escherichia coli cells; it is composed by three adjacent structural genes (plus some regulatory components): $l a c Z, l a c Y$ and $l a c A$ encoding for two enzymes $Z$ and $A$, and a transporter $Y$, involved in the digestion of the lactose. The main regulations are:

- the gene lacI encodes a repressor protein $I$;

- the DNA sequence, called promoter, is recognised by a RNA polymerase to iniziate the transcription of the genes $l a c Z, l a c Y$ and $l a c A$;

- a DNA segment, called the operator $(O P)$, obstructs the RNA polymerase functionality when the repressor protein $I$ is bound to it forming $I-O P$;

- a short DNA sequence, called the CAP-binding site, when it is bound to the complex composed by the protein $C A P$ and the signal molecule $c A M P$, acts as a promoter for the interaction between the RNA polymerase and the promoter.

The functionality of the lac operon depends on the integration of two control mechanisms, one mediated by lactose, and the other one mediated by glucose.

In the first control mechanism, an effect of the absence of the lactose is that $I$ is able to bind the operator sequence preventing the lac operon expression. If lactose is available, $I$ is unable to bind the operator sequence, and the lac operon can be potentially expressed.

In the second control mechanism, when glucose is absent, the molecule $c A M P$ and the protein $C A P$ increase the lac operon expression, thanks to the fact that the binding between the molecular complex $c A M P-C A P$ and the $C A P$-binding site increases. In summary, the condition promoting the operon gene expression is when the lactose is present and the glucose is absent.

In the following we report the description of the lac operon mechanism in the reaction system formalism and then show its encoding in cCNA. 


\subsection{The RS formalization}

The reaction system for the lac operon is defined as $A_{l a c}=(S, A)$, where the set $\mathrm{S}$ represents the main biochemical components involved in this genetic system, while the reaction set A contains the biochemical reactions involved in the regulation of the lac operon expression. Formally, the lac operon reaction system is defined as follows: $S$ is the set

$\{l a c, Z, Y, A, l a c I, I, I-O P, c y a, c A M P$, crp $, C A P, c A M P-C A P$, lactose, glucose $\}$,

and $\mathrm{A}$ consists of the following 10 reactions:

$$
\begin{array}{ll}
a_{1}=(\{l a c\},\{\ldots\},\{l a c\}), & a_{6}=(\{c y a\},\{\ldots\},\{c A M P\}), \\
a_{2}=(\{l a c I\},\{\ldots\},\{l a c I\}), & a_{7}=(\{\operatorname{lcrp}\},\{\ldots\},\{c r p\}), \\
a_{3}=(\{l a c I\},\{\ldots\},\{I\}), & a_{8}=(\{c r p\},\{\ldots\},\{C A P\}), \\
a_{4}=(\{I\},\{\text { lactose }\},\{I-O P\}), & a_{9}=(\{c A M P, C A P\},\{g l u c o s e\},\{c A M P-C A P\}), \\
a_{5}=(\{\text { lcya }\},\{\ldots\},\{c y a\}), & a_{10}=(\{\text { lac }, c A M P-C A P\},\{I-O P\},\{Z, Y, A\}) .
\end{array}
$$

The default context $(D C)$ is composed by those entities that are always present in the system $D C=\{l a c, l a c I, I, c y a, c A M P, \operatorname{crp}, C A P\}$, whereas the lactose and the glucose are given non-deterministically by the context.

\subsection{The RS encoding}

For the sake of readability, the encoding we propose exploits the specific features of the example in hand to perform some simplifications:

- for the entities in the default context, $s \in D C$, as they are persistent, we do not provide the $\overline{P_{s}}$ processes and the $C x t_{s}$ processes;

- for the reactions requiring the presence of entities $s \in D C$, we do not provide the reaction alternative behaviour when $s$ is absent;

- the $C x t_{s}$ processes are specified only for those entitiess that are really provided by the context.

Moreover, we do not model the dummy entity that is specified by dots (...) by the RS reactions in Section 5.2 Finally, we exclude the duplication reactions ( $a_{1}$, $a_{2}, a_{5}, a_{7}$ ), and renumber the remaining reactions :

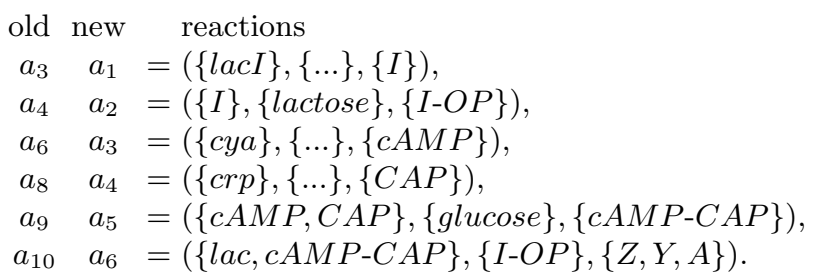

Expression reactions. First we define the parametric process

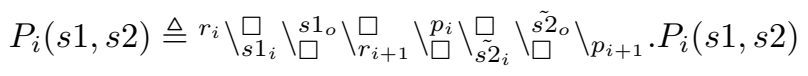

Then, we let $P_{a 1} \triangleq P_{1}(l a c I, I), P_{a 3} \triangleq P_{3}(c y a, c A M P)$, and $P_{a 4} \triangleq P_{4}(\operatorname{crp}, C A P)$. 
Regulation reactions.

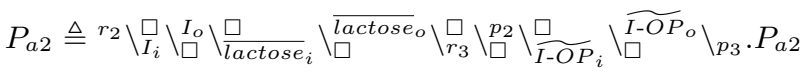

$$
\begin{aligned}
& +
\end{aligned}
$$

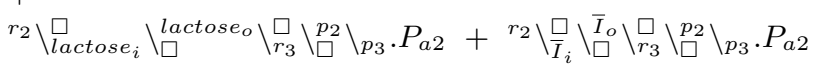

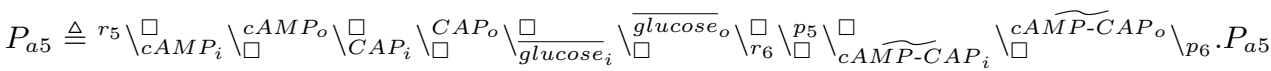

$$
\begin{aligned}
& + \\
& r_{5} \backslash g_{\text {glucose }} \backslash \stackrel{\text { glucose }}{\square} \backslash \backslash_{r_{6}}^{\square} \backslash_{\square}^{p_{5}} \backslash p_{6} . P_{a 5} \\
& +
\end{aligned}
$$

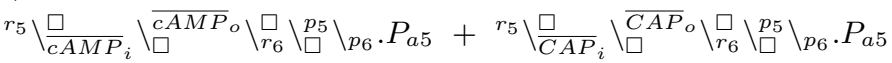

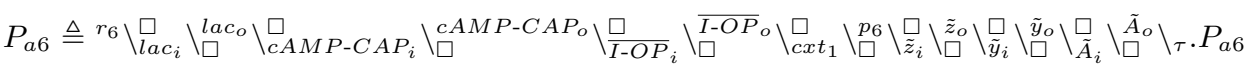

$$
\begin{aligned}
& + \\
& r_{6} \backslash_{I-O P_{i}}^{\square} \backslash_{\square}^{I-O P_{o}} \backslash_{c x t_{1}}^{\square} \backslash_{\square}^{p_{6}} \backslash_{\tau} \cdot P_{a 6} \\
& + \\
& r_{6} \backslash \frac{\square}{l_{a c}} \backslash_{\square}^{\overline{l a c}_{0}} \backslash_{c x t_{1}}^{\square} \backslash_{\square}^{p_{6}} \backslash_{\tau} \cdot P_{a 6}+\backslash \frac{\square}{c A M P-C A P_{i}} \backslash \backslash_{\square}^{\overline{c A M P-C A P}} \backslash_{c x t_{1}}^{\square} \backslash_{\square}^{p_{6}} \backslash_{\tau} \cdot P_{a 6}
\end{aligned}
$$

Processes for the entities. We exploit the specificity of the example in hand to optimise the code, and we specify exactly the number of solid links that each process encoding an entity must offer. For the always present entities we let:

$$
\begin{array}{ll}
P_{c y a} \triangleq c^{c y a_{i}} \backslash_{c y a_{o}} \cdot P_{c y a} & P_{c r p} \triangleq{ }^{c r p_{i}} \backslash_{c r p_{o}} \cdot P_{c r p} \\
P_{l a c I} \triangleq{ }^{l a c I_{i}} \backslash_{l a c I_{o}} \cdot P_{l a c I} & P_{l a c} \triangleq{ }^{l a c_{i}} \backslash_{l a c_{o}} \cdot P_{l a c}
\end{array}
$$

For the entities always produced (i.e. not present only at the first step), we provide a parametric definition $P_{e}(s) \triangleq s_{i} \backslash{ }_{s_{o}} \backslash \tilde{s}_{\square} \backslash \tilde{s}_{o} \cdot P_{e}(s)+\tilde{s}_{i} \backslash \tilde{s}_{o} \cdot P_{e}(s)$.

There are three entities of the second type:

$$
P_{C A M P} \triangleq P_{e}(c A M P) \quad P_{C A P} \triangleq P_{e}(C A P) \quad P_{I} \triangleq P_{e}(I) .
$$

The entity $I-O P$ can be either produced (by $a_{2}$ ) or tested for absence (by $\left.a_{6}\right)$. Correspondingly, the process $P_{I-O P}$ is defined as follows:

$$
\begin{aligned}
& P_{I-O P} \triangleq \sum_{h=0}^{1}\left({ }^{I-O P_{i}} \backslash_{I-O P_{o}}^{\square} \backslash\right)^{h \widetilde{I-O P}_{i}} \varlimsup_{\widetilde{I-O P} P_{o}} \cdot P_{I-O P}+{ }^{I-O P_{i}} \backslash_{I-O P_{o}} \cdot \overline{P_{I-O P}}
\end{aligned}
$$

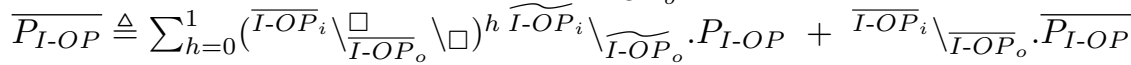

The process $P_{C A M P_{-} C A P}$ is similar to $P_{I-O P}$, as it is produced by $a_{5}$ and tested for presence by $a_{6}$. Its code is omitted.

The lactose is provided by the context and tested for absence by $a_{2}$. 


$$
\begin{aligned}
& P_{\text {lactose }} \triangleq \sum_{h=0}^{1}\left(\text { lactose }_{i} \backslash \text { lactose }_{o} \backslash \square\right)^{h \text { lactose }_{i}} \backslash_{\text {lactose }_{o}} \cdot P_{\text {lactose }} \\
& + \\
& \sum_{h=0}^{1}\left(\text { lactose }_{i} \backslash \backslash_{\text {lactose }_{o}} \backslash \square\right)^{h} \underline{\text { lactose }_{i}} \underline{\text { lactose }}_{\text {lactose }_{0}} \cdot \overline{P_{\text {lact }}}
\end{aligned}
$$

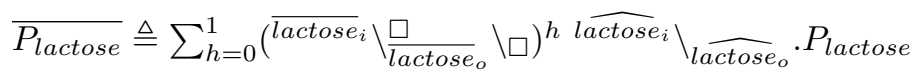

$$
\begin{aligned}
& + \\
& \sum_{h=0}^{1}\left(\overline{\text { lactose }}_{i} \backslash \frac{\square}{\text { lactose }_{o}} \backslash \square\right)^{h} \underline{\text { lactose }_{i}} \underline{\text { lactose }}_{\text {lactose }} . \overline{P_{\text {lact }}}
\end{aligned}
$$

The process $P_{\text {glucose }}$ is similar to $P_{\text {lactose }}$ and tested for absence by $a_{5}$. Its code is omitted.

The entity $z$ can only be produced by rule $a_{6}$, while it is never provided by the context. Moreover, there is no rule for testing its presence or absence.

$$
P_{z} \triangleq \tilde{z_{i}} \backslash \underset{z_{o}}{\square} \backslash \frac{z_{i}}{\square} \backslash \underline{z_{o}} \cdot P_{z}+\underline{z_{i}} \backslash \underline{z_{o}} \cdot \overline{P_{z}} \quad \overline{P_{z}} \triangleq \tilde{z_{i}} \backslash \underline{z_{o}} \backslash \backslash \frac{z_{i}}{\square} \backslash \underline{z_{o}} \cdot \overline{P_{z}}+\underline{z_{i}} \backslash \underline{z_{o}} \cdot \overline{P_{z}}
$$

The entities $y$ and $A$ are treated likewise $z$. Their processes are omitted.

Context. The entities in $D C$ are assumed always present by default, so no context process is needed for them. The entities $z, y$, and $A$ are assumed never provided by the context:

$$
\begin{aligned}
& C x t_{z} \triangleq c x t_{1} \backslash \underline{\underline{z}}_{i} \backslash \underline{\underline{z}}_{o} \backslash c x t_{2} \cdot C x t_{z} \quad C x t_{y} \triangleq c x t_{2} \backslash \underline{\underline{y}}_{i} \backslash \underline{\underline{y}}_{\square} \backslash c x t_{3} \cdot C x t_{y} \\
& C x t_{A} \triangleq c x t_{3} \backslash \underline{\square}_{i} \backslash \underline{\underline{A}}_{\square} \backslash c x t_{4} \cdot C x t_{A}
\end{aligned}
$$

Also, for the sake of presentation, we assume that the glucose is never provided and lactose is always provided by the context:

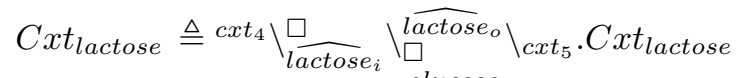

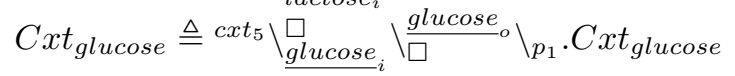

In the following we let $C X T \triangleq C x t_{z}\left|C x t_{y}\right| C x t_{A}\left|C x t_{\text {lactose }}\right| C x t_{\text {glucose }}$ be the processes for context. The whole system is as follows:

$$
l a c O p \triangleq(\nu \text { names })\left(\Pi_{i=1}^{6} P_{a i}\left|\Pi_{s \in D C} P_{s}\right| \Pi_{s \in S \backslash D C} \bar{P}_{s} \mid C X T\right)
$$

Execution. Now, we show the execution of two transitions. After the first transition, the entity $c A M P-C A P$ is produced due to the absence of glucose(see $\left.P_{a 5}\right)$, while the presence of lactose inhibits the production of $I$-OP (see $\left.P_{a 2}\right)$ : lacOp $\stackrel{(\nu \text { names }) v}{\longrightarrow}$ lacO $p^{\prime}$, where

$$
\begin{aligned}
v & ={ }^{\tau} \backslash_{\text {laci }_{i}} \ldots{ }^{\text {lactose }_{o}} \backslash_{r 3} \ldots{ }^{r_{5}} \backslash_{c A M P_{i}} \ldots{ }^{\overline{g l u c o s e}_{o}} \backslash_{r_{6}} \ldots{ }^{p_{5}} \backslash_{c A \widetilde{M P-C} A P_{i}} \ldots{ }^{p_{6}} \backslash_{\tau} \\
l a c O p^{\prime} & \triangleq(\nu \text { names })\left(\Pi_{i=1}^{6} P_{a i}\left|\Pi_{s \in A P} P_{s}\right| \Pi_{s \in S \backslash A P} \bar{P}_{s} \mid C X T\right)
\end{aligned}
$$

with $A P=D C \cup\{c A M P-C A P\}$ the actual context.

After the second step the entities $z, y$ and $A$ are produced, due to the presence of 
$c A M P-C A P$ and the absence of $I-O P\left(\right.$ see $\left.P_{a 6}\right)$, thus $l a c O p^{\prime} \stackrel{(\nu n a m e s) v^{\prime}}{\longrightarrow} l a c O p^{\prime \prime}$ where:

$v^{\prime}={ }^{\tau} \backslash_{l a c_{i}} \ldots{ }^{l a c_{o}} \backslash_{c A M P-C A P_{i}} \ldots{ }^{\overline{I-O P_{o}}} \backslash \ldots \ldots$ $l a c O p^{\prime \prime} \triangleq(\nu$ names $)\left(\Pi_{i=1}^{6} P_{a i}\left|\Pi_{s \in A P^{\prime}} P_{s}\right| \Pi_{s \in S \backslash A P^{\prime}} \bar{P}_{s} \mid C X T\right)$

with $A P^{\prime}=D C \cup\{z, y, A\}$.

\section{Enhanced Reaction Systems}

Our encoding increases the expressivity of RS concerning: the behaviour of the context, the possibility of alternative behaviour of mutated entities and the communication between two different reaction systems. It is important to note that our encoding guarantees that from each state, in the cCNA transition system, only one transition comes out, as the dynamics is totally deterministic.

\subsection{Recursive contexts}

In RS, the behaviour of the context is finite. For the first $n$ steps, it is specified which are the entities that are provided from the context. Using cCNA we can describe in a natural way the behaviour of the context in a recursive way. Then, the context behaviour would not necessarily end after $n$ steps, and could be infinite. For example, in an extended interactive process, we may want that the entity $s$ is intermittently provided by the context every two steps:

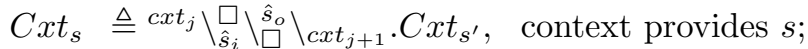

$$
\begin{aligned}
& C x t_{s^{\prime}} \triangleq c x t_{j} \backslash s_{s} \backslash\left\langle\underline{s}_{\square} \backslash{ }_{c x t_{j+1}} . C x t_{s^{\prime \prime}} \text {, context doesn't provide } s\right. \text {; } \\
& C x t_{s^{\prime \prime}} \triangleq c x t_{j} \backslash \underline{\underline{s}}_{i} \backslash \underline{\underline{s}}_{\square} \backslash c x t_{j+1} . C x t_{s}, \text { context doesn't provide } s \text {. }
\end{aligned}
$$

\subsection{Mutating entities}

In RS, when an entity is present, it can potentially be involved in each reaction where it is required. With a few more lines of code, in cCNA it is possible to describe the behaviour of a mutation of an entity, in a way that the mutated version of the entity can take part to only a subset of the rules requiring the normal version of the entity. For example, let us assume that entity $s 1$ is consumed by reactions $a 1$ and $a 2$. Reaction $a 1$ produces $s 1$ if $s 2$ is present, otherwise $a 1$ produces a mutated version of $s 1$, say $s 1^{\prime}$. When $s 1^{\prime}$ is produced, reaction $a 1$ behaves in the same way as if $s 1$ would be absent, whereas $a 2$ recognises the presence of $s 1^{\prime}$ and behaves in the same way as if $s 1$ would be present. Technically, in both cases it is enough to add one more non deterministic choice in the code of $P_{a 1}$ and $P_{a 2}$. 


$$
\begin{array}{|c|c|}
\hline r s 1 & r s 2 \\
\hline a_{1}=(s,, x) & a_{2}=(y,, s) \\
\hline
\end{array}
$$

Table 1: The two reaction systems $r s 1$ and $r s 2$.

\subsection{Communicating reaction systems}

We sketch how it is possible to program two RS encodings, in a way that the entities that usually come from context of one RS will be provided instead from the other RS.

Example 3. Let $r s 1$ and $r s 2$ be two RSs, defined by the rules in Table 1 . Now, we set our example such that the two contexts, for $r s 1$ and $r s 2$, do not provide any entities. We also assume that entity $s$ in $r s 1$ is provided by $r s 2$, as $r s 2$ produces a quantity of $s$ that is enough for $r s 1$ and $r s 2$. For technical reasons, we can not use the same name for $s$ in both the two RSs, then we use the name $s s$ in $r s 2$. We need to modify our translation technique to suite this new setting. As we do not model contexts, we introduce dummy channel names $d x$ and $d s s$ to model the case $x$ and $s s$ are not produced. Also, thanks to the simplicity of the example, we can leave out the use of the $p_{i}$ channels. This streamlining does not affect the programming technique we propose to make two RSs communicate. First, we translate the reaction in $r s 1$ :

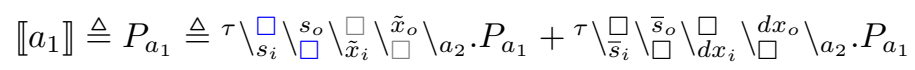

Please note, that prefixes of process $P_{a_{1}}$ end with the channel name $a_{2}$, as the link chain is now connected with the reaction of $r s 2$. The translation for the entities follows.

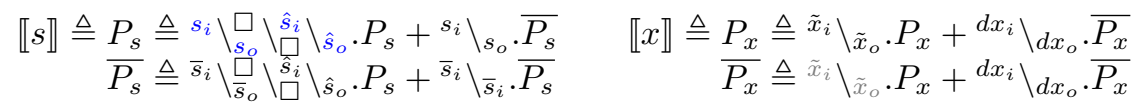

The translation for the $r s 2$ follows.

$$
\llbracket a_{2} \rrbracket \triangleq P_{a_{2}} \triangleq a_{2} \backslash \bigvee_{y_{i}}^{\square} \backslash_{\square}^{y_{o}} \backslash \backslash_{\tilde{s} s_{i}}^{\square} \backslash_{\square}^{\tilde{s} s_{o}} \backslash_{\tau} \cdot P_{a_{2}}+{ }^{a_{2}} \backslash \bar{y}_{i} \backslash \stackrel{\bar{y}}{\square}_{\square}^{\bar{y}_{d s s_{i}}} \stackrel{\square}{\square}^{d s s_{o}} \backslash_{\tau} \cdot P_{a_{2}}
$$

In the translation of the entities in $r s 2$, we introduce the mechanism that allows the entity $s$ ( $s s$ in $r s 2$ ) to be provided in $r s 1$. Every time $s s$ is produced in $r s 2$, a virtual link is created to synchronise with $r s 1$ on link ${ }^{\hat{s}_{i}} \backslash \hat{s}_{o}$ :

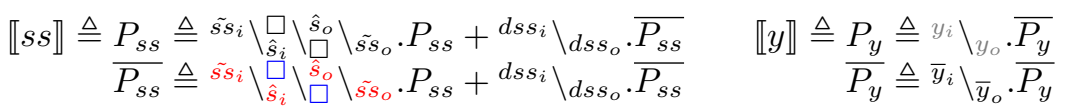

We now assume that the initial system is $S \triangleq(\nu$ names $)\left(P_{a_{1}}\left|P_{a_{2}}\right| P_{s}\left|P_{y}\right| \overline{P_{x}} \mid \overline{P_{s s}}\right)$, i.e. only entities $s$ and $y$ are present. Now, the only possible transition has the following label (that we report without restriction):

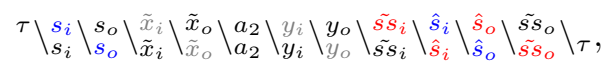


where the black links belong to the prefixes of $P_{a_{1}}$, and $P_{a_{2}}$, the blue links belong to $P_{s}$, the gray links belong to $P_{y}$, and $\overline{P_{x}}$ and the red links belong to $\overline{P_{s s}}$. After the execution, the entity $s$ is still present in $r s 1$ as it has been provided by $r s 2$.

As we have briefly sketched, our model of two communicating reaction systems can enable the study of the behaviour of one RS in relation to another one. Thus, the products of the reactions of one RS can become the input for another one. This could allow for a modular approach to modeling complex systems, by composing different Reaction Systems.

\section{Conclusion}

In this paper we have exploited a variant of the link-calculus where prefixes are link chains and no more single links. This variant was already briefly discussed by the end of $[8$. This variant allowed us to define an elegant embedding of reaction systems, an emerging formalism to model computationally biochemical systems. This translation shows several benefits. For instance, the context behaviour can also be expressed recursively; entity mutations can be expressed easily; reaction systems can communicate between them.

We believe that our embedding can contribute to extend the applications of reaction systems to diverse fields of computer science, and life sciences. As we have already mentioned, the evolution of each process resulting from our embedding is deterministic, thus we do not have the problem of having infinitely many transitions in the produced labelled transition system. In any case, we can exploit the implementation of the symbolic semantics of the link-calculus [1] that is available at $[19$.

As future work, we plan to implement a prototype of our framework, with an automatic translation from RSs to the link-calculus. We believe that our work can also help to extend the framework of RSs towards a model which can improve the communication between different RSs. We also believe that our work can make possible to investigate how to apply formal techniques to prove properties of the modeled systems [13/20/7/16]14].

Acknowledgments We thank the anonymous reviewers for their detailed and very useful criticisms and recommendations that helped us to improve our paper.

\section{References}

1. Azimi, S.: Steady states of constrained reaction systems. Theor. Comput. Sci. 701(C), 20-26 (2017). https://doi.org/10.1016/j.tcs.2017.03.047

2. Azimi, S., Iancu, B., Petre, I.: Reaction system models for the heat shock response. Fundamenta Informaticae 131(3-4), 299-312 (2014). https://doi.org/10.3233/FI2014-1016

3. Barbuti, R., Gori, R., Levi, F., Milazzo, P.: Investigating dynamic causalities in reaction systems. Theor. Comput. Sci. 623, 114-145 (2016) 
4. Bernini, A., Brodo, L., Degano, P., Falaschi, M., Hermith, D.: Process calculi for biological processes. Natural Computing 17(2), 345-373 (2018)

5. Bodei, C., Brodo, L., Bruni, R.: Open multiparty interaction. In: Recent Trends in Algebraic Development Techniques, 21st International Workshop, WADT 2012. Lecture Notes in Computer Science, vol. 7841, pp. 1-23. Springer (2012)

6. Bodei, C., Brodo, L., Bruni, R., Chiarugi, D.: A flat process calculus for nested membrane interactions. Sci. Ann. Comp. Sci. 24(1), 91-136 (2014)

7. Bodei, C., Brodo, L., Gori, R., Levi, F., Bernini, A., Hermith, D.: A static analysis for Brane Calculi providing global occurrence counting information. Theoretical Computer Science 696, 11-51 (2017)

8. Bodei, C., Brodo, L., Bruni, R.: A formal approach to open multiparty interactions. Theoretical Computer Science 763, 38-65 (2019)

9. Brijder, R., Ehrenfeucht, A., Main, M., Rozenberg, G.: A tour of reaction systems. International Journal of Foundations of Computer Science 22(07), 1499-1517 (2011)

10. Brodo, L.: On the expressiveness of pi-calculus for encoding mobile ambients. Mathematical Structures in Computer Science 28(2), 202-240 (2018)

11. Brodo, L., Olarte, C.: Symbolic semantics for multiparty interactions in the linkcalculus. In: Proc. of SOFSEM'17. Lecture Notes in Computer Science, vol. 10139, pp. 62-75. Springer (2017)

12. Cardelli, L., Gordon, A.D.: Mobile ambients. Theoretical Computer Science 240(1), 177-213 (2000)

13. Chiarugi, D., Falaschi, M., Hermith, D., Olarte, C., Torella, L.: Modelling nonmarkovian dynamics in biochemical reactions. BMC Systems Biology 9(S-3), S8 (2015)

14. Chiarugi, D., Falaschi, M., Olarte, C., Palamidessi, C.: Compositional modelling of signalling pathways in timed concurrent constraint programming. In: Proc.of ACM BCB'10. pp. 414-417. ACM, New York, NY, USA (2010)

15. Corolli, L., Maj, C., Marinia, F., Besozzi, D., Mauri, G.: An excursion in reaction systems: From computer science to biology. Theoretical Computer Science 454, 95-108 (2012)

16. Falaschi, M., Olarte, C., Palamidessi, C.: Abstract interpretation of temporal concurrent constraint programs. Theory and Practice of Logic Programming 15(3), 312-357 (2015)

17. Męski, A., Penczek, W., Rozenberg, G.: Model checking temporal properties of reaction systems. Information Sciences 313, 22-42 (2015). https://doi.org/10.1016/j.ins.2015.03.048

18. Okubo, F., Yokomori, T.: The computational capability of chemical reaction automata. Natural Computing 15(2), 215-224 (2016). https://doi.org/10.1007/s11047-015-9504-7

19. Olarte, C.: SiLVer: Symbolic links verifier (Dec 2018), http://subsell.logic.at/ links/links-web/index.html

20. Olarte, C., Chiarugi, D., Falaschi, M., Hermith, D.: A proof theoretic view of spatial and temporal dependencies in biochemical systems. Theor. Comput. Sci. 641, 25-42 (2016) 